\title{
DID YOU FEEL THAT? DEVELOPING NOVEL MULTIMODAL ALARMS FOR HIGH CONSEQUENCE CLINICAL ENVIRONMENTS
}

\author{
Parisa Alirezaee*, Roger Girgis*, TaeYong Kim*, Joseph J. Schlesinger ${ }^{\dagger}$, and Jeremy R. Cooperstock* \\ Centre for Interdisciplinary Research in Music, Media and Technology, Montreal, Canada \\ * Department of Electrical and Computer Engineering, McGill University, Montreal, Canada \\ ${ }^{\dagger}$ Depts. of Anesthesiology \& Biomedical Eng., Vanderbilt University Medical Center, Nashville, USA \\ \{parisa|rogerg|taeyong|jer\}@cim.mcgill.ca \\ joseph.j.schlesinger@vanderbilt.edu
}

\begin{abstract}
Hospitals are overwhelmingly filled with sounds produced by alarms and patient monitoring devices. Consequently, these sounds create a fatiguing and stressful environment for both patients and clinicians. As an attempt to attenuate the auditory sensory overload, we propose the use of a multimodal alarm system in operating rooms and intensive care units. Specifically, the system would utilize multisensory integration of the haptic and auditory channels. We hypothesize that combining these two channels in a synchronized fashion, the auditory threshold of perception of participants will be lowered, thus allowing for an overall reduction of volume in hospitals. The results obtained from pilot testing support this hypothesis. We conclude that further investigation of this method can prove useful in reducing the sound exposure level in hospitals as well as personalizing the perception and type of the alarm for clinicians.
\end{abstract}

\section{INTRODUCTION}

The operating room (OR) and intensive care unit (ICU) are noisy environments, exacerbated by frequent auditory alarms. As an example, the average sound level of patient rooms in three Veterans Affairs facilities were measured at $51 \mathrm{~dB}(\mathrm{~A})$ [1], whereas the World Health Organization (WHO) recommends sound levels 15$20 \mathrm{~dB}(\mathrm{~A})$ lower to avoid negative impact on patients and staff, such as sleep deprivation and alarm fatigue.

The high incidence of alarms in the ICU and OR command attention, raise stress, and are often irrelevant to the responsibilities of individual clinicians. In multi-bed care areas in hospitals, one can count more than 30 different alarm sounds [2]. The increasing number of alarms in hospitals cause problems because of the lack of clinical information provided by their interfaces and the stressful environment caused by their overall volume. Although nurses and doctors rely on patient-monitoring devices for diagnosis and treatment, a high number of these devices increase the rate of false alarms by not reflecting a medically urgent condition. This, in turn, may lead to error-prone situations.

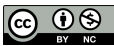

This work is licensed under Creative Commons Attribution Non Commercial 4.0 International License. The full terms of the License are available at http://creativecommons.org/licenses/by-nc/4.0
Additionally, loudness of the alarms can cause "alarm fatigue", which is a phenomenon of diminished response due to desensitization of the practitioners [3]. This problem is exacerbated by the fact that free-field audible alarms are not informative, nonlocalizable, and presented to everyone in the room. When the fatigue is severe, it can increase clinician error, potentially leading to adverse patient outcomes.

To cope with these problems, we are interested in the possibility of leveraging multimodality to improve the information transfer capacity of alarms. Through this approach to alarm management, we anticipate a reduction in overall sound exposure level in the clinical environment and decreased distractions, as well as a possibility of implementing personalized alarms.

An extensive volume of previous literature has described the effects of multisensory integration, in particular involving the auditory and visual modalities. These include evidence for both complementary and inhibitory effects of the combination [4]. However, visual attention is directional, and in the case of a clinical environment, one cannot assume that a visual signal would be attended to by the health-care provider. In contrast, haptic feedback can be provided anywhere, any time, irrespective of current activity, and offers the additional benefit that it can be delivered selectively to the clinician(s) for whom it is relevant.

To investigate the possibility of leveraging the audio-haptic modality in this manner, we conducted an experiment to determine the degree to which haptic stimuli can complement audible alarms. To quantify the accuracy to such cues, we compared unisensory auditory and multisensory auditory-haptic stimuli.

The results of our experiments did not demonstrate a facilitative effect as expected. However, analyzing them raised important questions regarding fatigue and habituation to vibrotactile stimuli, potential interference of sensory streams, potential benefits of speech over non-speech auditory stimuli, and if supra-threshold stimuli can still be weakly effective as a unisensory stream to contribute to multisensory gain.

\section{LITERATURE REVIEW}

To understand the importance of studying alarm systems in the hospital setting, Block divides the set of problems with audible alarms into several categories: 1) false alarms; 2) loud alarms; 3) difficulty in determining the device that is making the alarm sound; and 4) inability to stop an alarm [5]. 
These problems worsen with the fact that devices designed by different manufacturers may use different alarm sounds to indicate the same event occurrence. Block then discusses how an alarm sound can address a specific medical situation. His approach grouped audible alarms into a set of six different sounds, each related to one tissue injury situation for the patient, and assigned a different priority. He tested different melodies for these alarm sounds as well as the training method to instruct the clinicians to determine the sounds and their meanings.

In a similar vein, Edworthy focuses on false alarm events in hospitals [3] [6], investigating important issues related to the design, implementation, classification, and standardization of medical alarms. Although the design of medical alarms may seem trivial, the literature makes clear that despite the large investment in the development of standards, i.e., IEC 60601-1-8 [7], significant problems remain. For example, Talley et al. found a false alarm rate of $85 \%$ to $99 \%$ in cardiopulmonary monitors [8]. Unfortunately, this level of false alarms has not changed significantly over the past 25 years. As another approach Keller tackles the false alarm problem by reducing more generally the number of alarms step-by-step to reach an acceptable level [9]. For each step, a safety assessment analyzed the response process to a task on alarm notification. This approach succeeded in reducing the number of alarms from a high number to around four per day.

The aforementioned issues are recognized by regulatory bodies. In the 2015 top 10 Health Technology Hazard list published by the Emergency Care Research Institute (ECRI), the top priority was stated to be clinical alarm hazards. Nurses spend an unnecessary amount of time on alarm management and on dealing with ambiguous alarm implementations. According to the U.S. Food and Drug Administration (FDA), in the span of four years, more than 500 patient deaths have been associated with faulty alarm management [10]. Despite these issues, audible alarms tend to be the most effective method in attracting the attention of practitioners when they are occupied with other tasks.

However, as audible alarms became the prominent method, their use had the unintended consequence of Alarm Fatigue [10]. Without a formal operational definition, this phenomenon can be understood as the desensitization to alarm sounds as a result of hearing too many alarms at the same time. Thus, the medical staff is incapable of responding to alarms with equal urgency.

With multiple alarm stimuli, there is attentional competition and auditory competition (e.g., masking). Hasanain et al. explored the issue of simultaneous masking; a condition where because of the interaction between concurrent sounds, one or more of them become imperceptible due to the physical limitation of human perception [11]. According to the Pennsylvania Patient Safety Authority, 194 documented problems with operators responses to alarms over a span of four years resulted in twelve deaths [12]. The cause was found to be related to the number of simultaneous alarms sounding in the medical environment. Detecting alarm masking is highly challenging as it may occur only in a very specific interaction of multiple sounds. Hasanain et al. proposed a method to do so without experimentation [11]. Instead, the authors used psychophysical modeling in the configuration of medical alarms, which builds on previous work done by Hasanain and Bolton [13].

The problem of "Alarm Fatigue" has been investigated by understanding the psychoacoustic properties of alarms and analyzing the sensory perception of clinicians. Ongoing experiments by Schlesinger et al. [14] are designed to determine the auditory per- ceptual threshold of alarms while participants perform other attentional demanding audiovisual tasks. Hospital alarms are typically louder than background noise, i.e., a positive signal-to-noise ratio. However, their results showed that clinician performance measured in response time and accuracy was preserved when alarms were softer than background noise, i.e., a negative signal-to-noise ratio.

The method we propose in the present work attempts to employ multisensory integration; specifically, in combining the audio and haptic channels, with the goal of reducing the sound exposure level, and therefore stress level, in the hospital environment. The Principle of Inverse Effectiveness (PoIE) [15] suggests that an enhanced neural response can be achieved when stimuli from two modalities are simultaneously presented. This effect becomes greater if the stimuli produce a weak response when presented unimodally. Experiments have shown this perceptual additivity at sub-threshold levels from neural inputs of the olfactory and gustatory channels [16]. Furthermore, co-occurrence of a sound was found to increase accuracy and enhance the sensitivity for detection of near-threshold visual stimuli [17].

The PoIE led us to theorize that the co-occurrence of a haptic stimulus and an audio stimulus would allow participants to perceive sound below their audio threshold of perception. In the OR and ICU setting, this would allow a reduction of the alarm intensity while maintaining the effectiveness in cueing a staff member. Furthermore, it may be advantageous to present the haptic stimulus at a sub-threshold level to not disrupt the procedure being performed by the prompted medical professional. Visell et al. [18] showed that the addition of sub-threshold stimuli affected the participants perception of compliance. This provides encouraging evidence that even at a sub-threshold level, such stimuli may be sufficient to affect alarm perception.

\section{METHODOLOGY}

There is evidence from the literature to support the hypothesis that multisensory integration may lead to participants perceiving sound at a lower threshold. However, we are equally interested in determining whether this effect may hold when the non-audio stimulus is delivered at a sub-threshold level. That is, can we reduce the level of auditory alarms in a clinical environment by delivering a complementary non-auditory stimulus, ideally, one that the clinician does not even perceive?

In order to investigate this question, it was first necessary to determine the unimodal thresholds of perception for both the auditory and non-auditory stimuli. Our experiment therefore consists of three measurements:

\section{1. haptic (vibration) perception threshold}

2. auditory perception threshold

3. auditory perception threshold when combined with haptic stimulus

One of the most popular methods to map the relationship between physical stimuli and psychological response of the participant [19] is Parameter Estimation by Sequential Testing (PEST) [20], [21], an adaptive staircase method that has shown its adaptability and robustness in obtaining a perceptual threshold value. Figure 1 represents a typical double staircase for measuring the auditory threshold for one of the participants. An improvement is to double the step size in response to several identical responses, 


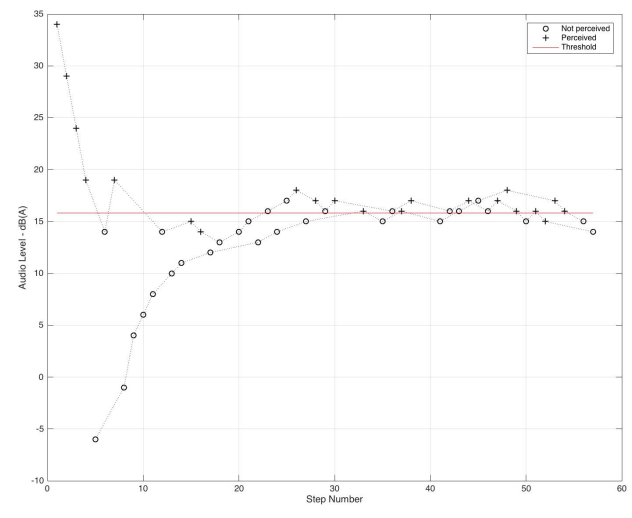

Figure 1: An example of the double-staircase method to determine the auditory threshold of perception for one of the experiment participants.

and halve the step size in response to a change in consecutive responses. This helps achieve faster convergence and improves participant focus, and was therefore adopted for our testing [19].

To reduce the effects of bias that arises after several identical responses to a given stimulus, Cornsweet suggested the use of the random double-staircase method [22]. The test participant is presented with two staircases, starting from values above and below the assumed threshold, respectively. The step size begins relatively high to ensure fast convergence, and as the two staircases approach each other, the step size is reduced to ensure a smooth combination of the two. This results in a range of values bounding the threshold of perception.

Throughout our experiment, we employ the PEST procedure coupled with the use of the random double staircase to determine the threshold of perception.

\section{EXPERIMENTS}

\subsection{Environment}

The experiment was performed in the Centre for Interdisciplinary Research in Music Media and Technology (CIRMMT). The lab is acoustically insulated from the surrounding rooms. In addition, participants wore Beyerdynamic DT 770 Pro (Heilbronn, Germany) circumaural headphones during the experiment, and the ambient temperature was maintained between 21 and 25 throughout the tests, thereby ensuring a well-controlled environment.

\subsection{Stimuli}

To provide the vibrotactile stimulus, we used a Tactile Labs Haptuator Mark I (Montreal, Canada) [23], which allows for independent variation of the amplitude and the frequency. For our experiment, the haptuator was connected to a Sparkfun TP2005D1 audio amplifier (Boulder, CO, USA), and strapped snugly to the participants' leg, above the ankle, using a Velcro band. The choice of placing the vibrational device on the leg rather than the wrist was motivated by our intended use case of delivering alarm signals in a medical environment, for which hygienic constraints preclude the wearing of devices on the hands or wrists. A potential confound is that actuation of the vibrational device may be audible, contributing to the sound volume of the alarm stimulus. However, this was mitigated by the low intensity of vibration, the placement of the actuator on the participants' leg, and the use of closed headphones throughout the experiment.

Delivery of stimulus during the experiment and logging of measurements for the double staircase was managed by a MATLAB script (MathWorks MATLAB R2016a, Natick, MA, USA). A one-second auditory stimulus was extracted from a recording of the Philips MP-70 (Amsterdam, Netherlands) patient monitor $\mathrm{red} / \mathrm{crisis}$ alarm. The frequency spectrum of the alarm sound is shown in Figure 2. The choice of a one-second duration was deemed to be reasonably short to help eliminate guesses, and sufficiently long so as to include the salient auditory characteristics of the alarm signal.

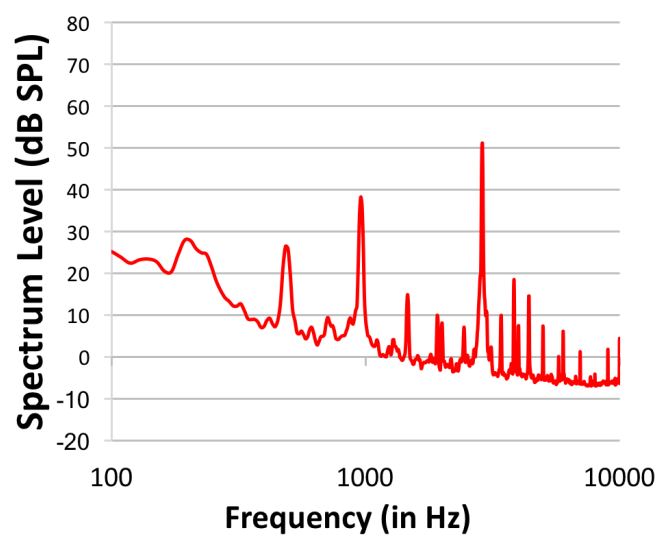

Figure 2: Alarm waveform, measured with a class II Amprobe SM20A sound level meter provided a weighted output of $49 \mathrm{~dB}$.

The vibratory stimulus was generated using a sine wave at 175 $\mathrm{Hz}$, output using the MATLAB sound() function for a duration of 1 second. For the combined auditory-haptic stimuli, the signals were output in unison, using a stereo audio splitter to separate the audio (left channel) and haptic (right channel) signals.

\subsection{Experimental Procedure}

Participants first completed a pretest questionnaire to screen for possible health conditions that might exclude them from the experiment. They were then asked to read an instruction sheet, put on the headphones, and assisted with securing of the haptic band just above the ankle at a comfortable location, as shown in Figure 3.

The strap was secured in a snug fashion for good coupling between the actuator and the skin, but not so tight that it caused discomfort. Participants were then asked to place their right foot in a comfortable position and to immobilize it for the remainder of the experiment.

Participants then proceeded through the first block of the experiment, which determined their haptic perceptual threshold. In the second block, we determined the auditory perceptual threshold, both with and without a combined haptic stimulus. This was done by intermingling two tests, randomly selecting half of the trials for presentation of unimodal auditory stimuli, and half for presentation of combined audio-haptic stimuli with the haptic stimuli 


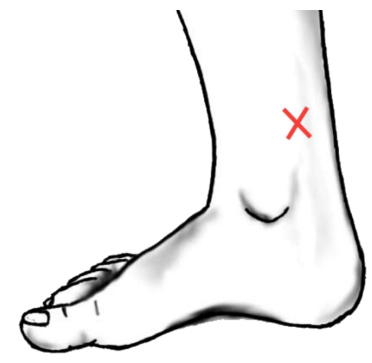

Figure 3: Position placement of the actuator on the participants' ankle, from experiment instructions.

delivered at a fixed level. This intermingling was done to avoid potential habituation effects that may have biased the threshold estimates in either direction.

For each experimental condition, participants initially carried out a training/calibration step to familiarize themselves with the experimental stimuli and adjust these to a level in which they were barely perceptible, using a coarse staircase method. Subsequent to this initial adjustment, the PEST method, using a dual-staircase procedure, was employed to determine the participants' perceptual thresholds. Each stimulus was delivered randomly within an $8 \mathrm{~s}$ window following the previous stimulus.

To ensure that participants did not go too long between successive perceivable stimuli, which we observed during pilot testing as a significant source of fatigue, the system randomly delivered $20 \%$ of the stimuli at 3.5 standard deviations above the average of the intensities of the last six stimuli, a choice we determined empirically as adequate to ensure reasonable supra-threshold perception.

For our purposes, participants had to respond within $2 \mathrm{~s}$ from the onset of the stimulus, i.e., no more than $1 \mathrm{~s}$ following its presentation, by clicking on a button displayed in a simple graphical user interface; otherwise, it was assumed that they did not perceive the stimulus. The system then reduced or increased the subsequent stimulus intensity by a defined step size so as to maintain the intensity just at the edge of perceptibility. The step size was reduced as both staircases converged, i.e., as the difference in intensities between the upward and downward staircases decreased. This process continued until a minimum step size was reached, then six reversals were counted on each staircase for the threshold estimation. The perceptual threshold was estimated as the mean of the stimulus levels of the last six reversals from each staircase.

The aforementioned design was employed for all of our experiments, with variations as described in the following sections.

\subsection{Pilot}

Initially, we fixed the sub-threshold amplitude of vibration at 2.5 standard deviations below the perceptual threshold determined in the first block.

Pilot testing was performed on 11 lab members (10 male, 1 female), over the span of three days. The mean age of the participants was 30.4 years with a standard deviation of 8.6 years. Data from one of these participants were excluded from the analysis for failing to respond to supra-threshold "wake-up stimuli", which suggested a lack of attention during the experiment. The test participants participated on a voluntary basis and did not receive monetary compensation for their time.
Five of the 10 participants whose data were retained for analysis exhibited a slightly lower auditory threshold when measured in the multimodal audio-haptic condition than in the unimodal audioonly condition. Although the results of these initial tests were only borderline in terms of statistical significance $(\mathrm{p}=0.15, \mathrm{ci}=[-0.74$, $0.13]$ ), we were encouraged to carry out a larger experiment with additional participants who were naive to the experimental hypothesis.

\subsection{Full Experiment}

The same experimental procedure was then applied to a new group of participants, naive as to the experimental hypothesis. These participants were not informed that sub-threshold vibration was delivered (in conjunction with half of the audio stimuli) during the second block. The test was conducted on 12 participants (10 male, 2 female), over the span of three days. The mean age of the participants was 28 years with standard deviation of 3.5 years.

Data from one of these participants, exhibiting a difference between the audio-only and audio-haptic thresholds greater than six times, were excluded from the analysis as an outlier. The test participants participated on a voluntary basis and received monetary compensation for their time. The auditory threshold values measured across 12 participants for both the audio-only condition and the audio-haptic condition is shown in Figure 4. The differences between the conditions are shown in the bar graph of Figure 5.

As can be seen, the data did not support our hypothesis that the perceptual threshold is reduced in the multimodal condition. Counter-intuitively, the trend suggested an opposite effect, although not significant $(\mathrm{p}=0.78, \mathrm{ci}=[-0.65,0.83])$. This led us to consider the possibility that our haptic stimuli was too far below the perceptual threshold, and was thus not contributing to the effect.

\subsection{Increased Haptic Intensity Level}

To address the possibility that the haptic stimuli were too far below threshold to have an impact, we then conducted a further experiment in which the amplitude of vibration was increased to 0.5 standard deviations below the threshold determined in block 1 . As before, participants were not informed that they might feel vibrations during the third block.

Ten participants ( 4 male, 6 female) were recruited and the test took place during one day. The mean age of the participants was 27.3 years with standard deviation of 5.7 years. Data collection from one of the participants could not be completed on account of the participant changing the computer's output volume in the middle of the test. This participant was excluded from the analysis. The test participants participated in the study on a voluntary basis and received monetary compensation for their time.

The perception threshold in the auditory domain measured across 9 participants for both the unisensory and multisensory conditions is shown in Figure 6. The differences between the conditions are shown in the bar graph of Figure 7.

By way of response to a post-test questionnaire, 5 out of 9 participants indicated that on occasion, they perceived the haptic stimulus during the second block.

Despite the increase of the level of haptic stimulus, we found no statistically significant difference between the threshold of audio perception in the unisensory and multisensory conditions $(\mathrm{p}=$ $0.21, \mathrm{ci}=[-0.90,0.23])$. 


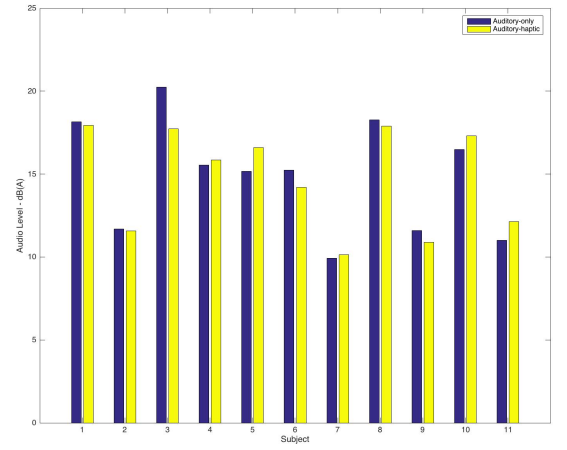

Figure 4: Threshold data obtained from the audio-haptic and audio-only threshold measurement over the 11 participants whose data were retained.

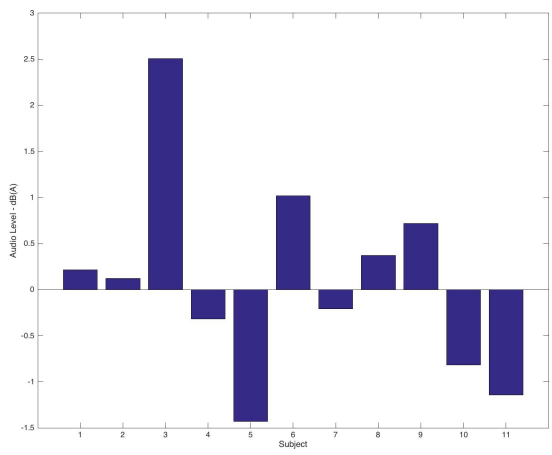

Figure 5: Differences between audio-only and audio-haptic threshold values from Figure 4. A positive value indicates that the auditory perceptual threshold was reduced in the multimodal condition.

\section{CIRCLING AROUND THE THRESHOLDS}

We considered several possibilities for the results of the previous section:

1. Either or both auditory and haptic perception thresholds varied throughout the experiment, e.g., due to fatigue or habituation, and so the thresholds measured in blocks 1 and 2 were unreliable.

2. The presentation of simultaneous sub- or near-threshold haptic stimuli interfered with auditory perception.

3. There is no multimodal integration benefit from haptic stimuli in conjunction with non-speech audio.

4. There is a multimodal integration benefit from haptic stimuli in conjunction with non-speech audio, but only for supra-threshold haptic stimuli.

To examine these possibilities, we conducted an additional exploratory experiment, in which we varied the level of auditory stimuli in a range of \pm 2 standard deviations and varied the level of haptic stimuli in a range of $[-2,+4]$ standard deviations around the unimodal thresholds determined in blocks 1 and 2 .

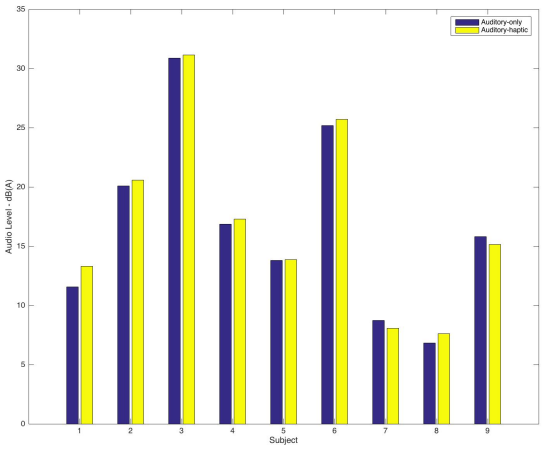

Figure 6: Threshold data obtained from the audio-haptic and audio-only threshold measurement over the 9 participants from the increased haptic intensity level experiment.



Figure 7: Differences between audio-only and audio-haptic threshold values from Figure 6 . A positive value indicates that the auditory perceptual threshold was reduced in the multimodal condition.

The results of this exploration were revealing, although hardly conclusive: three of the five participants, two of whom were coauthors of this paper demonstrated no discernible effect of haptic stimulus on audio stimulus detection, even at clearly suprathreshold levels of haptic stimuli. The remaining two participants demonstrated a possible effect, with slightly higher rates of audio alarm detection for sub-threshold audio when presented in conjunction with supra-threshold haptic stimuli (see Figure 8 for the results of one of these participants). However, it does not appear that these results are significant, and thus, we can neither confirm nor reject any of the possibilities described at the start of this section. 


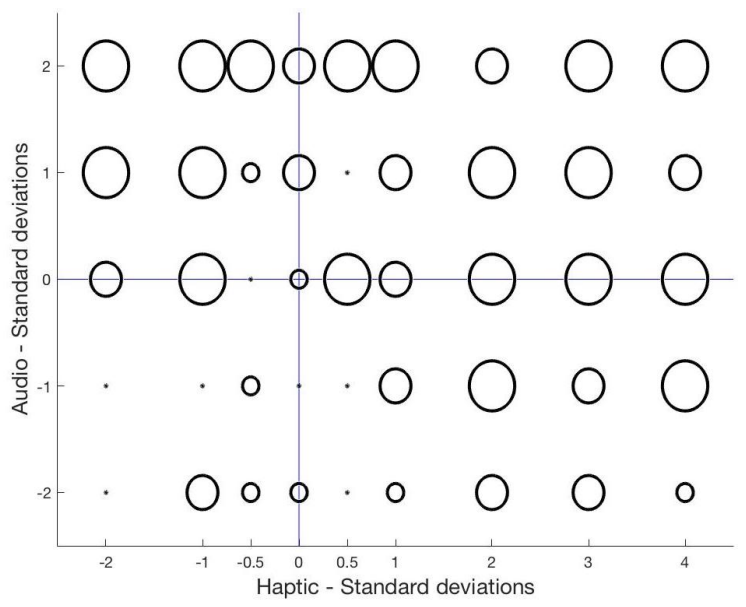

Figure 8: Detection rate for one participant of audio stimuli as a function of audio and haptic stimulus level, relative to the thresholds measured in blocks 1 and 2. The size of each circle indicates the detection rate (out of three presentations) at each combination of parameters.

\section{CONCLUSIONS AND FUTURE DIRECTIONS}

We postulated at the outset of this research that improved perception of auditory stimuli would result through multimodal integration with a complementary haptic signal, possibly even at subthreshold levels. If so, we hypothesized that this could allow for attenuation of alarm fatigue and assist the practitioner in recognizing the alarm, thereby reducing the problems of stress and alarm fatigue in the clinical settings of the OR and ICU.

Through these experiments, we hoped to determine preliminary guidelines for the outcomes of implementing multimodal alarms, leading to a reduction in the demands on the audio channel. We hypothesize that a multimodal alarm system can attenuate alarm fatigue and assist the practitioner in recognizing the alarm.

While we have so far not been able to verify this hypothesis, we believe that the experimental protocol we developed to address the research questions here will prove valuable to the multisensory research community and can be applied to future experiments that seek to resolve some of the unanswered questions raised in Section 5. It is also possible that the PoIE, as observed in other experimental contexts, is only manifested in conjunction with speech auditory stimuli, for which the neurophysiological responses are differently affected by the influence of a secondary stimulus modality. This would need to be determined through a separate experiment, employing a speech cue rather than an auditory alarm sound.

Future experiments can employ the PoIE by adding hospital background noise, auditory speech-in-noise tasks, and visual vigilance tasks to test if the hypotheses would still hold while testing clinicians during simulated emergencies requiring clinical pharmacologic intervention. These data will not only inform alarm design and improve patient safety, but have wide-ranging applications to other high consequence industries.

\section{ACKNOWLEDGMENT}

Colleagues in the Shared Reality Lab, in particular, Francesco Tordini and Jeffrey Blum, provided several rounds of helpful feedback and constructive comments throughout this research, and the technical staff at the Centre for Interdisciplinary Research in Music Media and Technology (CIRMMT) offered invaluable assistance with our experiments. The authors are most grateful to these individuals. The authors also wish to thank the Vanderbilt University Medical Center, Department of Anesthesiology (especially Drs. Matthew Weinger and Pratik Pandharipande). Vanderbilt University Department of Hearing and Speech Sciences (especially Drs. Wesley Grantham, Ben Hornsby, and Dan Ashmead).

\section{REFERENCES}

[1] M. H. Otenio, E. Cremer, and E. M. T. Claro, "Noise level in a 222 bed hospital in the 18th health region-pr," Revista Brasileira de Otorrinolaringologia, vol. 73, no. 2, pp. 245250, 2007.

[2] J. H. Kerr, "Warning Devices," British journal of anaesthesia, vol. 57, no. 7, pp. 696-708, 1985.

[3] J. Edworthy, "Medical audible alarms: a review," Journal of the American Medical Informatics Association, vol. 20, no. 3, pp. 584-589, 2013.

[4] D. Burr, M. S. Banks, and M. C. Morrone, "Auditory dominance over vision in the perception of interval duration," $E x$ perimental Brain Research, vol. 198, no. 1, p. 49, 2009.

[5] F. E. Block, "For if the trumpet give an uncertain sound, who shall prepare himself to the battle?" Anesthesia and Analgesia, vol. 106, no. 2, pp. 357-359, 2008.

[6] J. R. Edworthy, J. J. Schlesinger, R. R. McNeer, M. S. Kristensen, and C. L. Bennett, "Classifying alarms: Seeking durability, credibility, consistency, and simplicity," Biomedical Instrumentation \& Technology, vol. 51, no. s2, pp. 50-57, 2017.

[7] "Medical electrical equipment, part 18: General requirements, tests and guidance for alarm systems in medical electrical equipment and medical electrical systems. iec 606011-8." International Electrotechnical Commission, 2006.

[8] L. B. Talley, J. Hooper, B. Jacobs, C. Guzzetta, R. McCarter, A. Sill, S. Cain, and S. L. Wilson, "Cardiopulmonary monitors and clinically significant events in critically ill children," Biomedical Instrumentation \& Technology, vol. 45, no. s1, pp. 38-45, 2011.

[9] J. P. Keller, R. Diefes, K. Graham, M. Meyers, and K. Pelczarski, "Why clinical alarms are a top ten hazard: how you can help reduce the risk," Biomedical Instrumentation \& Technology, vol. 45, no. s1, pp. 17-23, 2011.

[10] M. S. Kristensen, J. Edworthy, and S. Denham, "Alarm fatigue in the perception of medical soundscapes," European Congress and Exposition on Noise Control Engineering, pp. 745-750, 2015.

[11] B. Hasanain, A. D. Boyd, J. Edworthy, and M. L. Bolton, "A formal approach to discovering simultaneous additive masking between auditory medical alarms," Applied Ergonomics, vol. 58, pp. 500-514, 2017.

[12] P. P. Advisory, "Connecting remote cardiac monitoring issues with care areas." Pa Patient Saf Authority, vol. 6, no. 3, pp. 79-83, 2009. 
[13] B. Hasanain, A. D. Boyd, and M. L. Bolton, "An approach to model checking the perceptual interactions of medical alarms," in Proceedings of the Human Factors and Ergonomics Society Annual Meeting, vol. 58, no. 1. SAGE Publications, 2014, pp. 822-826.

[14] J. J. Schlesinger, "Utilizing multisensory integration to improve psychoacoustic alarm design in the intensive care unit."

[15] M. A. Meredith and B. E. Stein, "Visual, auditory, and somatosensory convergence on cells in superior colliculus results in multisensory integration," Journal of neurophysiology, vol. 56, no. 3, pp. 640-662, 1986.

[16] J. Diamond, P. A. Breslin, N. Doolittle, H. Nagata, and P. Dalton, "Flavor processing: perceptual and cognitive factors in multi-modal integration," Chemical senses, vol. 30, no. suppl 1, pp. i232-i233, 2005.

[17] T. Noesselt, S. Tyll, C. N. Boehler, E. Budinger, H.-J. Heinze, and J. Driver, "Sound-induced enhancement of lowintensity vision: multisensory influences on human sensoryspecific cortices and thalamic bodies relate to perceptual enhancement of visual detection sensitivity," Journal of Neuroscience, vol. 30, no. 41, pp. 13 609-13 623, 2010.

[18] Y. Visell, B. L. Giordano, G. Millet, and J. R. Cooperstock, "Vibration influences haptic perception of surface compliance during walking," PLoS one, vol. 6, no. 3, p. e17697, 2011.

[19] S. A. Gelfand and H. Levitt, Hearing: An introduction to psychological and physiological acoustics. Marcel Dekker New York, 1998, vol. 4.

[20] M. Taylor and C. D. Creelman, "Pest: Efficient estimates on probability functions," The Journal of the Acoustical Society of America, vol. 41, no. 4A, pp. 782-787, 1967.

[21] M. Taylor, S. Forbes, and C. D. Creelman, "Pest reduces bias in forced choice psychophysics," The Journal of the Acoustical Society of America, vol. 74, no. 5, pp. 1367-1374, 1983.

[22] T. N. Cornsweet, "The staircase-method in psychophysics," The American journal of psychology, vol. 75, no. 3, pp. 485491, 1962.

[23] H.-Y. Yao and V. Hayward, "Design and analysis of a recoiltype vibrotactile transducer," The Journal of the Acoustical Society of America, vol. 128, no. 2, pp. 619-627, 2010. 